\title{
Ground State Properties of Charmed Hypernuclei with Mean Field Approach
}

\author{
H. Güven \\ Université Paris-Saclay, CNRS/IN2P3, IJCLab, 91405 Orsay, France and \\ Physics Department, Yildiz Technical University, 34220 Esenler, Istanbul, Turkey \\ K. Bozkurt \\ Physics Department, Yildiz Technical University, 34220 Esenler, Istanbul, Turkey and \\ Université Paris-Saclay, CNRS/IN2P3, IJCLab, 91405 Orsay, France \\ E. Khan \\ Université Paris-Saclay, CNRS/IN2P3, IJCLab, 91405 Orsay, France
}

\author{
J. Margueron \\ Univ Lyon, Univ Claude Bernard Lyon 1, CNRS/IN2P3, \\ IP2I Lyon, UMR 5822, F-69622, Villeurbanne, France
}

(Dated: June 23, 2021)

\begin{abstract}
Closed shell charmed hypernuclei ${ }_{\Lambda_{c}^{5}}^{5} \mathrm{Li},{ }_{\Lambda_{c}}^{17} \mathrm{~F},{ }_{\Lambda_{c}}^{41} \mathrm{Sc},{ }_{\Lambda_{c}}^{57} \mathrm{Cu},{ }_{\Lambda_{c}}^{133} \mathrm{Sb}$ and ${ }_{\Lambda_{c}}^{209} \mathrm{Bi}$ are calculated within Hartree-Fock approach by using three different force sets derived from microscopic BruecknerHartree-Fock calculations of $\Lambda$ hypernuclei. Ground state properties (binding energies, $\Lambda_{c}$ separation energies, $\Lambda_{c}$ single particle energies and $\Lambda_{c}$ densities) of charmed nuclei are examined. Due to the Coulomb repulsion between protons and the $\Lambda_{c}$ baryon, charmed hypernuclei are most bound for $16 \leq \mathrm{A} \leq 41$, where ${ }_{\Lambda_{c}}^{17} \mathrm{~F}$ can be considered as an excellent candidate to measure charmed hypernuclei. The competition between the attractive nucleon- $\Lambda_{c}$ interaction and the Coulomb repulsion is discussed, and we compare $\Lambda$ and $\Lambda_{c}$ hypernuclei properties.
\end{abstract}

\section{INTRODUCTION}

The discovery of charmed hadron $\Lambda_{c}[1-6]$, after the one of the (strange) $\Lambda$ hyperon [7], opened the possibility to produce charmed hypernuclei, an extension of very exotic hypernuclei. From a theory viewpoint, such systems were first discussed in the seminal work of Dover and Kahana in 1977 [8], where bound charmed nuclei were predicted, based on an interaction potential generated by SU(4) symmetry. Meantime, experimental efforts to investigate charmed nuclei were performed in Dubna in the 1970's and 1980's [9-11]. In these experiments, only three candidate were found, namely $\Lambda_{c} \mathrm{Li}, \Lambda_{c} \mathrm{~B}$ and $\Lambda_{c} \mathrm{~N}$, where the separation energy of $\Lambda_{c}$ was measured to be between 0 and $10 \mathrm{MeV}$ [11]. In the future, GSI-FAIR (Gesellschaft für Schwerionenforschung-Facility for Antiproton and Ion Research) and JPARC (Japan Proton Accelerator Research Complex) facilities are expected to produce sufficient charmed particles to generate more charmed hypernuclei [12-14].

The key ingredient in the theoretical description of charmed hypernuclei is the nucleon- $\Lambda_{c}\left(\mathrm{~N} \Lambda_{c}\right)$ interaction. Historically, SU(4) flavor symmetry was considered in order to determine $\mathrm{N} \Lambda_{c}$ potential, similarly to the phenomenological nucleon-nucleon $(\mathrm{NN})$ and $\mathrm{N} \Lambda$ potentials. With such potentials, the ground state properties were obtained using few-body methods [15-19]. However this method reaches its limits as the nuclear mass number grows, and it becomes more relevant to consider density functional theory (DFT) approaches. In the past few years, charmed hypernuclei have been revisited, based on DFT, e.g. relativistic mean field (RMF) model [20, 21], quark meson coupling (QMC) model [2225], or the Brueckner-Hartree-Fock approach [26]. The results obtained from these DFT approaches differ, especially because of their different $\mathrm{N} \Lambda_{c}$ interaction. For instance, the coupling constants between charmed baryons and mesons are usually obtained from the quark counting rules in QMC and RMF models. Another approach for describing $\mathrm{N} \Lambda_{c}$ potential, is using lattice QCD simulation: the central part and the tensor part of $\mathrm{N} \Lambda_{c}$ interaction are calculated by HAL QCD Collaboration [27]. It is found that $\Lambda_{c}$ hypernuclei can exist between $\mathrm{A}=12$ to $\mathrm{A} \approx 50$ [27]. Then, Haidenbauer and Krein extrapolated the $\mathrm{N} \Lambda_{c}$ interaction from the HAL QCD one, with physical pion mass and chiral effective field theory [28]. Vidaña et al. also calculated charmed hypernuclei, using $\mathrm{SU}(4)$ extension of the meson-exchange hyperon-nucleon potential of the Jülich group, where phase shifts of Model $\mathrm{B}$ and Model $\mathrm{C}$ are compatible with the low energy region of extrapolation from Haidenbauer and Krein [26]. Recently, charmed hypernuclei from ${ }_{\Lambda_{c}^{5}}^{5} \mathrm{Li}$ to ${ }_{\Lambda_{c}}^{209} \mathrm{Bi}$ have been calculated with the same approach developed by Haidenbauer et al. [29]. On the contrary to HAL QCD results [27], both Vidaña et al. and Haidenbauer et al. (see Refs. [26, 29] for details) predicted bound $\Lambda_{c}$ hypernuclei for the $\mathrm{A} \geq 50$ region, where $\Lambda_{c}$ binding energies are compatible with previous QMC calculations [22-24]. The $\Lambda_{c}$ bound states of QMC calculations are inside the gap defined between model $\mathrm{A}$ and Model $\mathrm{C}$ of Vidaña et al. for the $\mathrm{A} \leq 50$ region. However, the interactions calculated from the HAL QCD with the chiral effective field theory [29] results in a less attractive $\mathrm{N} \Lambda_{c}$ potential compared to the interactions derived from hyperon- 
nucleon potential of the Jülich group [26], where the binding energy differences are $7.2 \pm 1.5 \mathrm{MeV}$ for Model $\mathrm{C}$ and $49 \pm 1.5 \mathrm{MeV}$ for Model A for ${ }_{\Lambda_{c}}^{209} \mathrm{Bi}$ charmed hypernucleus. Another recent calculation was done by $\mathrm{Wu}$ et al., with QMC model, where coupling constants are obtained from both the naive quark counting rules and the HAL QCD predictions [25]. They confirm the results of Vidaña et al. [26] for the coupling constant obtained from the naive quark counting rules, where bound $\Lambda_{c}$ states are calculated up to ${ }_{\Lambda_{c}}^{209} \mathrm{~Pb}$. However, they also find compatible result with HAL QCD Collaboration [27] from the coupling constant obtained from HAL QCD simulation, where bound $\Lambda_{c}$ states are only possible for the $\mathrm{A} \leq 52$ region.

In this work, we explore the ground state properties of charmed hypernuclei with non-relativistic DFT approach, where the $\mathrm{N} \Lambda_{c}$ interaction is fixed within the DFT framework. In the Skyrme-Hartree-Fock framework, the N $\Lambda$ channel is based on a G-matrix calculation starting from various bare interactions: NSC89, NSC97a and NSC97f (Nijmegen Soft Core Potentials) [30]. The density functional, deduced from the G-matrix calculation in uniform matter, will be generically named as DF-NSC89, DF-NSC97a and DF-NSC97f, hereafter, see Ref. [31] for more details. The oldest DF-NSC89 functional can reproduce with a good accuracy the experimental single particle energies of $\Lambda$ hyperon for light hypernuclei such as ${ }_{\Lambda}^{5} \mathrm{He}$ or ${ }_{\Lambda}^{13} \mathrm{C}$, but for the heavier hypernuclei like ${ }_{\Lambda}^{41} \mathrm{Ca}$ or ${ }_{\Lambda}^{209} \mathrm{~Pb}$, DF-NSC97a and DF-NSC97f give results close to the experimental data $[30,32]$. In order to generate $\mathrm{N} \Lambda_{c}$ interaction, we use the approach introduced by Starkov et al., where the similarity of the in-medium interaction between $\mathrm{N} \Lambda_{c}$ and $\mathrm{N} \Lambda$ channels is considered [33]. We detail this scheme in the next section.

It should be noted that the notation of charmed nuclei is changing in the literature, whether the charge of $\Lambda_{c}^{+}$is considered or not. In this work, we consider the chemical convention, where the element name refers to the total charge of the nucleus (including protons and $\Lambda_{c}^{+}$charges), as well as the mass is the total mass counting nucleons and hyperons). In the following, we will investigate ${ }_{\Lambda_{c}^{5}}^{5} \mathrm{Li}$ which can be decomposed as $\left[{ }^{4} \mathrm{He}+\Lambda_{c}\right]$, as well as ${ }_{\Lambda_{c}} \mathrm{~F}$ $\left(\left[{ }^{16} \mathrm{O}+\Lambda_{c}\right]\right),{ }_{\Lambda_{c}}^{41} \mathrm{Sc}\left(\left[{ }^{40} \mathrm{Ca}+\Lambda_{c}\right]\right),{ }_{\Lambda_{c}} \mathrm{Cu}\left(\left[{ }^{56} \mathrm{Ni}+\Lambda_{c}\right]\right),{ }_{\Lambda_{c}}^{133} \mathrm{Sb}$ $\left(\left[{ }^{132} \mathrm{Sn}+\Lambda_{c}\right]\right),{ }_{\Lambda_{c}}{ }^{209} \mathrm{Bi}\left(\left[{ }^{208} \mathrm{~Pb}+\Lambda_{c}\right]\right)$. Such nuclei have been selected in the present study since the nucleon cores are all closed shell, which allows to neglect deformation and pairing effects. They are expected to be almost spherical, and the possible small deformation coming from $\Lambda_{c}$ is neglected because of the doubly neutron and proton closed shells. We therefore consider Equal Filling Approximation (EFA) for the occupation probability of the 1s $\Lambda_{c}$-states [34]. Finally, we shall investigate binding energies, single particle spectra, Coulomb repulsion and density distribution of $\Lambda_{c}$, in related charmed hypernuclei.

The paper is organized in the following way: Sec. II, details the Skyrme-Hartree-Fock framework and the cal- culation of $\mathrm{N} \Lambda_{c}$ interaction. In Sec. III, the ground state properties of charmed hypernuclei are discussed. Finally, a brief summary and some concluding remarks are given in Sec. IV.

\section{THEORETICAL FRAMEWORK}

Considering a non-relativistic system, composed of interacting nucleons $N$ and $\Lambda_{c}^{\prime} s$, the total Hamiltonian reads,

$$
\widehat{H}=\widehat{T}_{N}+\widehat{T}_{\Lambda_{c}}+\widehat{H}_{N N}+\widehat{H}_{N \Lambda_{c}},
$$

where $\widehat{T}_{N}\left(\widehat{T}_{\Lambda_{c}}\right)$ are the kinetic energy operators for nucleons $\left(\Lambda_{c}^{\prime} s\right)$ and $\widehat{H}_{N N}\left(\widehat{H}_{N \Lambda_{c}}\right)$ are the interaction operator terms acting between $N$ and $N\left(N\right.$ and $\left.\Lambda_{c}\right)$ species.

\section{A. Mean-field approximation}

In the mean field approximation, the ground state of the system is the tensor product $\left|\Psi_{N}\right\rangle \otimes\left|\Psi_{\Lambda}\right\rangle$, where $\left|\Psi_{N}\right\rangle$ $\left(\left|\Psi_{\Lambda}\right\rangle\right)$ is a slater determinant of the nucleon $\left(\Lambda_{c}\right)$ states. The total Hamiltonian (1) can be turned into a density functional $\epsilon\left(\rho_{N}, \rho_{\Lambda_{c}}\right)$, function of the particle densities $\rho_{N}$ and $\rho_{\Lambda_{c}}$, as $\widehat{H}=\int \epsilon\left(\rho_{N}, \rho_{\Lambda_{c}}\right) d^{3} r$. The energy functional $\epsilon$ is often expressed as [32, 35],

$$
\begin{aligned}
\epsilon\left(\rho_{N}, \rho_{\Lambda_{c}}\right)= & \frac{\hbar}{2 m_{N}} \tau_{N}+\frac{\hbar}{2 m_{\Lambda_{c}}} \tau_{\Lambda_{c}}+\epsilon_{N N}\left(\rho_{N}\right) \\
& +\epsilon_{N \Lambda_{c}}\left(\rho_{N}, \rho_{\Lambda_{c}}\right)+\epsilon_{\mathrm{Coul}}\left(\rho_{p}, \rho_{\Lambda_{c}}\right)
\end{aligned}
$$

where $\tau_{N}\left(\tau_{\Lambda_{c}}\right)$ is the nucleonic (charmed hyperonic) kinetic energy density, $\epsilon_{\text {Coul }}\left(\rho_{p}, \rho_{\Lambda_{c}}\right)$ is the Coulomb interaction which depends on proton and $\Lambda_{c}$ densities, and $\epsilon_{N N}$ and $\epsilon_{N \Lambda_{c}}$ are the interaction terms of the energy density functional describing the $\mathrm{NN}$ and $\mathrm{N} \Lambda_{c}$ channels.

In the following, the nucleonic terms will be deduced from the well known SLy5 Skyrme interaction [36], widely used for the description of the structure of finite nuclei. In order to calculate the $N \Lambda_{c}$ interaction channel, we use a scaling relation introduced by Starkov et. al., [33] and defined as,

$$
\epsilon_{N \Lambda_{c}}=K \epsilon_{N \Lambda}
$$

where the $K$ factor is 0.8 , from the estimation of coupling constant of $\sigma$ and $\omega$ mesons. Accordingly, the $N \Lambda$ interaction is given by a density functional $\epsilon_{N \Lambda}$, adjusted to BHF predictions in uniform matter [32, 35],

$$
\epsilon_{N \Lambda}\left(\rho_{N}, \rho_{\Lambda}\right)=-f_{1}\left(\rho_{N}\right) \rho_{N} \rho_{\Lambda}+f_{2}\left(\rho_{N}\right) \rho_{N} \rho_{\Lambda}^{5 / 3}
$$

In the $N \Lambda$ channel, the spin-orbit doublets are experimentally undistinguishable, typically around 100-200 $\mathrm{KeV}[37,38]$, and the spin-orbit interaction among $\Lambda$ particles can safely be neglected [39]. An interesting mechanism, based on the quark sub-structure of hadrons 
TABLE I: Parameters of the functionals DF-NSC89, DF-NSC97a and DF-NSC97f.

\begin{tabular}{lllllll}
\hline \hline Force & $\alpha_{1}$ & $\alpha_{2}$ & $\alpha_{3}$ & $\alpha_{4}$ & $\alpha_{5}$ & $\alpha_{6}$ \\
\hline DF-NSC89 & 327 & 1159 & 1163 & 335 & 1102 & 1660 \\
DF-NSC97a & 423 & 1899 & 3795 & 577 & 4017 & 11061 \\
DF-NSC97f & 384 & 1473 & 1933 & 635 & 1829 & 4100 \\
\hline \hline
\end{tabular}

explains the strong reduction of the spin-orbit in the $N \Lambda$ channel [40]. Even smaller spin-orbit splitting could be expected for $N \Lambda_{c}$ channel, due to the large mass of $\Lambda_{c}$ baryons $\left(m_{\Lambda_{c}}=2286.46 \mathrm{MeV}\right.$, see Ref. [41] for details) $[14,40]$. Therefore, we also omit spin-orbit interaction in the case of the $N \Lambda_{c}$ interaction.

We now recall the details of the $N \Lambda$ channel, where the following density functionals are considered: DFNSC89 [35], DF-NSC97a [32], DF-NSC97f [32]. The functions $f_{1-2}$ in Eq. (4) are expressed as,

$$
\begin{aligned}
& f_{1}\left(\rho_{N}\right)=\alpha_{1}-\alpha_{2} \rho_{N}+\alpha_{3} \rho_{N}^{2}, \\
& f_{2}\left(\rho_{N}\right)=\alpha_{4}-\alpha_{5} \rho_{N}+\alpha_{6} \rho_{N}^{2},
\end{aligned}
$$

where $\alpha_{1-6}$ are constants given in Table I.

In uniform nuclear matter, the single particle energies read,

$$
\epsilon_{N}(k)=\frac{\hbar^{2} k^{2}}{2 m_{N}^{*}}+v_{N}^{\mathrm{matt}} \text { and } \epsilon_{\Lambda}(k)=\frac{\hbar^{2} k^{2}}{2 m_{\Lambda}^{*}}+v_{\Lambda}^{\mathrm{matt}},
$$

where the potentials $v_{N}$ and $v_{\Lambda}$ derive from the energy functional as

$$
\begin{aligned}
& v_{N}^{\text {matt }}\left(\rho_{N}, \rho_{\Lambda}\right)=v_{N}^{\text {Skyrme }}+\frac{\partial \epsilon_{N \Lambda}}{\partial \rho_{N}}, \\
& v_{\Lambda}^{\text {matt }}\left(\rho_{N}, \rho_{\Lambda}\right)=\frac{\partial \epsilon_{\Lambda \Lambda}}{\partial \rho_{\Lambda}}+\frac{\partial \epsilon_{N \Lambda}}{\partial \rho_{\Lambda}} .
\end{aligned}
$$

In the case of hypernuclei, the energy functional defined in Eq. (4) is corrected by the effective mass term as (see Ref. [42] and therein),

$$
\begin{aligned}
& \epsilon_{N N}^{\text {nucl }}=\epsilon_{N N}-\frac{3 \hbar^{2}}{10 m_{N}} \rho_{N}^{5 / 3}\left(\frac{6 \pi^{2}}{g_{N}}\right)^{2 / 3}\left[\frac{m_{N}}{m_{N}^{*}}-1\right], \\
& \epsilon_{N \Lambda}^{\text {nucl }}=\epsilon_{N \Lambda}-\frac{3 \hbar^{2}}{10 m_{\Lambda}} \rho_{\Lambda}^{5 / 3}\left(\frac{6 \pi^{2}}{g_{\Lambda}}\right)^{2 / 3}\left[\frac{m_{\Lambda}}{m_{\Lambda}^{*}}-1\right],
\end{aligned}
$$

where the effective mass correction for the $\mathrm{N} \Lambda$ part can be expressed as a polynomial in the nucleonic density $\rho_{N}$ as [35],

$$
\frac{m_{\Lambda}^{*}\left(\rho_{N}\right)}{m_{\Lambda}}=\mu_{1}-\mu_{2} \rho_{N}+\mu_{3} \rho_{N}^{2}-\mu_{4} \rho_{N}^{3} .
$$

The values for the parameters $\mu_{1-4}$ are given in Tab. II. The effective mass correction of nucleon is given from Skyrme interaction [43].
TABLE II: The parameters of the $\Lambda$-effective mass.

\begin{tabular}{lllll}
\hline \hline Force & $\mu_{1}$ & $\mu_{2}$ & $\mu_{3}$ & $\mu_{4}$ \\
\hline DF-NSC89 & 1.00 & 1.83 & 5.33 & 6.07 \\
DF-NSC97a & 0.98 & 1.72 & 3.18 & 0 \\
DF-NSC97f & 0.93 & 2.19 & 3.89 & 0 \\
\hline \hline
\end{tabular}

TABLE III: Parameters of the functionals DF-NSC89-C, DF-NSC97a-C and DF-NSC97f-C.

\begin{tabular}{lllllll}
\hline \hline Force & $\alpha_{1}^{c}$ & $\alpha_{2}^{c}$ & $\alpha_{3}^{c}$ & $\alpha_{4}^{c}$ & $\alpha_{5}^{c}$ & $\alpha_{6}^{c}$ \\
\hline DF-NSC89-C & 261.6 & 927.2 & 930.4 & 268 & 881.6 & 1328 \\
DF-NSC97a-C & 338.4 & 1519.2 & 3036 & 461.6 & 3213.6 & 8848.8 \\
DF-NSC97f-C & 307.2 & 1178.4 & 1546.4 & 508 & 1463.2 & 3280 \\
\hline \hline
\end{tabular}

\section{B. Charmed hypernuclei}

We now generate the $\Lambda_{c}$ interaction for charmed hypernuclei, employing Eq. (3), which transforms the $\mathrm{N} \Lambda$ force into a $\mathrm{N} \Lambda_{c}$ force:

$$
\epsilon_{N \Lambda_{c}}\left(\rho_{N}, \rho_{\Lambda_{c}}\right)=-f_{1}\left(\rho_{N}\right) \rho_{N} \rho_{\Lambda_{c}}+f_{2}\left(\rho_{N}\right) \rho_{N} \rho_{\Lambda_{c}}^{5 / 3},
$$

where the functions $f_{1-2}$ are

$$
\begin{aligned}
& f_{1}\left(\rho_{N}\right)=\alpha_{1}^{c}-\alpha_{2}^{c} \rho_{N}+\alpha_{3}^{c} \rho_{N}^{2}, \\
& f_{2}\left(\rho_{N}\right)=\alpha_{4}^{c}-\alpha_{5}^{c} \rho_{N}+\alpha_{6}^{c} \rho_{N}^{2},
\end{aligned}
$$

where $\alpha_{1-6}^{c}$ are new constants for $\Lambda_{c}$. These constants are given in Table III, corresponding to $\mathrm{K}=0.8$ in Eq. (3). Hereafter we call these force sets as DF-NSC89-C, DFNSC97a-C and DF-NSC97f-C. However, since nothing is known about effective mass for $\Lambda_{c}$, we use the same effective mass parameters than for the $\mathrm{N} \Lambda$ interaction defined in Eq. (12). Finally the energy functional for $\mathrm{N} \Lambda$ interaction becomes,

$$
\epsilon_{N \Lambda_{c}}^{\text {nucl }}=\epsilon_{N \Lambda_{c}}-\frac{3 \hbar^{2}}{10 m_{\Lambda_{c}}} \rho_{\Lambda_{c}}^{5 / 3}\left(\frac{6 \pi^{2}}{g_{\Lambda_{c}}}\right)^{2 / 3}\left[\frac{m_{\Lambda}}{m_{\Lambda}^{*}}-1\right] .
$$

Since $\Lambda_{c}$ is a positively charged particle, one needs to include Coulomb interaction to the total energy functional defined in Eq.(2). Coulomb interaction is decomposed in direct and exchange terms as,

$$
\epsilon_{\text {Coul }}\left(\rho_{p}, \rho_{\Lambda_{c}}\right)=E_{\text {Coul }}^{D}+E_{\text {Coul }}^{E} .
$$

The direct Coulomb term is,

$$
E_{\text {Coul }}^{D}=\sum_{i \neq j} \frac{e^{2}}{2} \int d^{3} \mathbf{r} d^{3} \mathbf{r}^{\prime} \rho_{i}(\mathbf{r}) \frac{1}{\left|\mathbf{r}-\mathbf{r}^{\prime}\right|} \rho_{j}\left(\mathbf{r}^{\prime}\right),
$$

where $i, j=p, \Lambda_{c}$. It should be noted that the $p \Lambda_{c}$ channel is repulsive with respect of direct Coulomb interaction. 
Considering the Slater approximation, the exchange term reads,

$$
E_{\text {Coul }}^{E}=-e^{2} \frac{3}{4}\left(\frac{3}{\pi}\right)^{\frac{1}{3}} \int d^{3} \mathbf{r}\left(\rho_{p}^{4 / 3}+\rho_{\Lambda_{c}}^{4 / 3}\right) .
$$

The exchange term is attractive for all charged particles.

\section{Hartree-Fock equations}

We are now ready to extend our calculation to generate ground state properties of charmed hypernuclei. The spherically symmetric Hartree-Fock (HF) framework is considered for present single-charmed hypernuclei, close to doubly magic nuclei. In the HF approach, the Schrödinger equation can be obtained by minimizing the total energy defined in Eq. (2), and using Skyrme model for the nucleonic part [36], The usual Schrödinger equation is then obtained $\left(i=N, \Lambda_{c}\right)$,

$$
\begin{array}{r}
{\left[-\nabla \cdot \frac{\hbar^{2}}{2 m_{i}^{*}(r)} \nabla+V_{i}(r)-i W_{i}(r)(\nabla \times \sigma)\right] \psi_{i, \alpha}(r)} \\
=-e_{i, \alpha} \psi_{i, \alpha}(r),
\end{array}
$$

where $V_{i}$ is the interaction potential and $W_{i}$ is the spin-orbit potential [44] which is naturally obtained by Skyrme model [36]. Since the spin-orbit interaction is neglected for $\Lambda_{c}$ channel, $W_{\Lambda_{c}}=0$ in Eq. (20).

The interaction potentials are extracted by taking the functional derivative of the energy with respect to the densities. Following the DFT framework, the nucleon potential reads

$$
\begin{array}{r}
V_{N}(\mathbf{r}) \equiv v_{N}^{\text {Skyrme }}+\frac{\partial \epsilon_{N \Lambda_{c}}}{\partial \rho_{N}}+\frac{\partial}{\partial \rho_{N}}\left[\frac{m_{\Lambda}}{m_{\Lambda}^{*}\left(\rho_{N}\right)}\right] \\
\times\left[\frac{\tau_{\Lambda_{c}}}{2 m_{\Lambda_{c}}}-\frac{3}{5} \frac{\left(3 \pi^{2}\right)^{2 / 3} \hbar^{2}}{2 m_{\Lambda_{c}}} \rho_{\Lambda_{c}}^{5 / 3}\right] \\
\quad+v_{\text {Coul }, \mathrm{p}}^{D}(r)+v_{\text {Coul }, \mathrm{p}}^{E}(r),
\end{array}
$$

and the $\Lambda_{c}$ potential is given by

$$
\begin{aligned}
V_{\Lambda_{c}}(\mathbf{r}) \equiv \frac{\partial \epsilon_{N \Lambda_{c}}}{\partial \rho_{\Lambda_{c}}}-[ & \left.\frac{m_{\Lambda}}{m_{\Lambda}^{*}\left(\rho_{N}\right)}-1\right] \frac{\left(3 \pi^{2}\right)^{2 / 3} \hbar^{2}}{2 m_{\Lambda_{c}}} \rho_{\Lambda_{c}}^{2 / 3} \\
& +v_{\mathrm{Coul}, \Lambda_{c}}^{D}(r)+v_{\mathrm{Coul}, \Lambda_{c}}^{E}(r),
\end{aligned}
$$

where $v_{\mathrm{Coul}, i}^{D}(r) \quad\left(v_{\mathrm{Coul}, i}^{E}(r)\right)$ is the direct (exchange) Coulomb potential, which can be extracted by taking derivatives for related density $\rho_{i}\left(i=\mathrm{p}, \Lambda_{c}\right)$. Direct Coulomb potential is

$$
v_{\mathrm{Coul}, i}^{D}(r)=e^{2} \int d^{3} \mathbf{r}^{\prime} \frac{1}{\left|\mathbf{r}-\mathbf{r}^{\prime}\right|} \rho_{c h}\left(\mathbf{r}^{\prime}\right),
$$

where $\rho_{c h}=\rho_{p}+\rho_{\Lambda_{c}}$ is the charge density. We consider the extension of the Slater approximation for multiple types of charged particles, giving for the exchange Coulomb potential

$$
v_{\text {Coul }, i}^{E}(r)=-e^{2}\left(\frac{3}{\pi}\right)^{1 / 3} \int d^{3} \mathbf{r}^{\prime}\left[\rho_{i}\left(\mathbf{r}^{\prime}\right)\right]^{1 / 3} .
$$
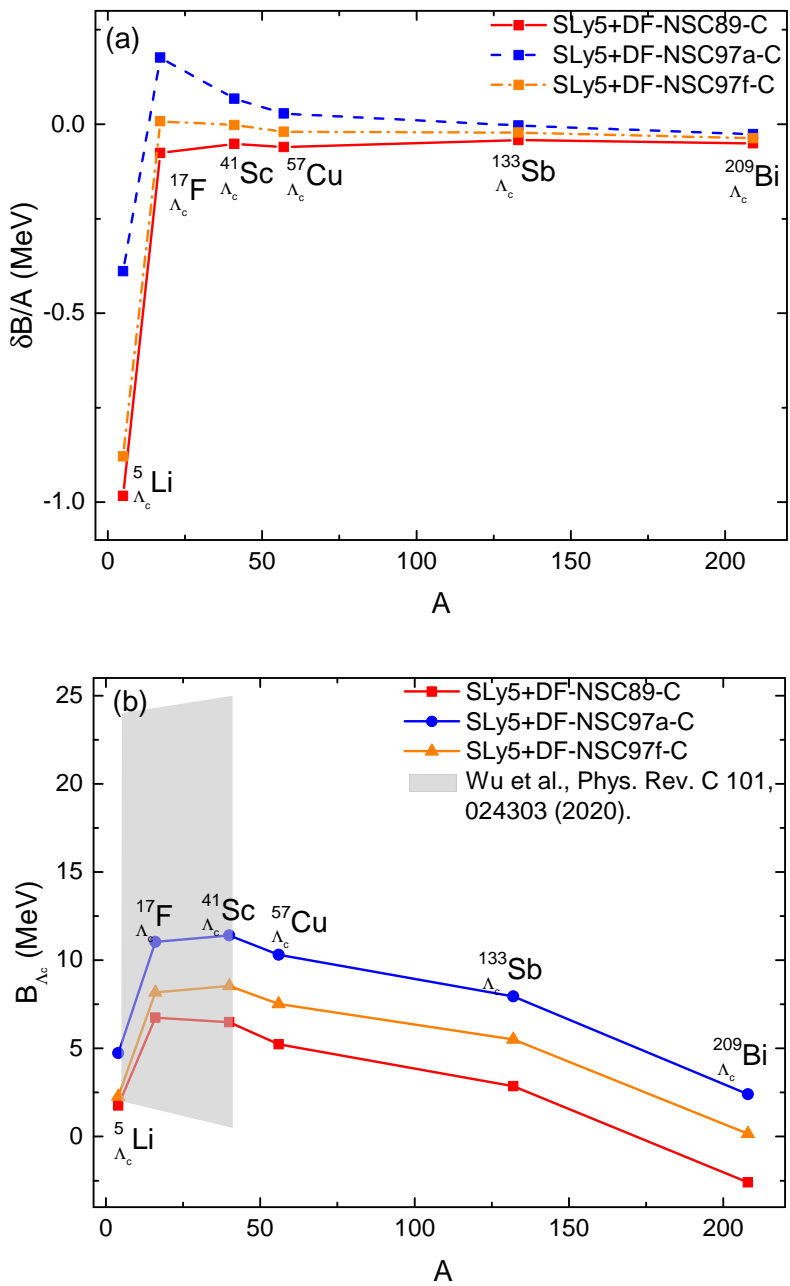

FIG. 1: (a) The difference on binding energy per baryon $\delta B / A$, with respect to one of the nucleus without the charmed baryon, and (b) the $\Lambda_{c}$ separation energy $B_{\Lambda_{c}}$ for DF-NSC89-C, DF-NSC97a-C and DF-NSC97f-C force sets.

where $i=\mathrm{p}, \Lambda_{c}$. As mentioned above, the direct Coulomb potential is always repulsive while the exchange Coulomb potential is always attractive in the case of charmed hypernuclei.

\section{RESULTS}

\section{A. The Ground State Properties of $\Lambda_{c}$ Hypernuclei}

In this section, starting from the lightest charmed hypernuclei ${ }_{\Lambda_{c}^{5}}^{5} \mathrm{Li}$, we discuss the ground state properties of ${ }_{\Lambda_{c}}^{17} \mathrm{~F},{ }_{\Lambda_{c}}^{41} \mathrm{Sc},{ }_{\Lambda_{c}}^{57} \mathrm{Cu},{ }_{\Lambda_{c}}^{133} \mathrm{Sb}$ and ${ }_{\Lambda_{c}}^{209} \mathrm{Bi}$

The difference on binding energy per baryon $\delta B / A=$ $B / A\left({ }_{\Lambda_{c}}^{\mathrm{A}+1} Z+1\right)-B / A\left({ }^{\mathrm{A}} \mathrm{Z}\right)$, and the $\Lambda_{c}$ separation energy $B_{\Lambda_{c}}=E\left({ }_{\Lambda_{c}}^{\mathrm{A}+1} Z+1\right)-E\left({ }^{\mathrm{A}} \mathrm{Z}\right)$, are calculated for 
$\Lambda_{c}^{5} \mathrm{Li},{ }_{\Lambda_{c}}^{17} \mathrm{~F},{ }_{\Lambda_{c}}^{41} \mathrm{Sc},{ }_{\Lambda_{c}}^{57} \mathrm{Cu},{ }_{\Lambda_{c}}^{133} \mathrm{Sb}$ and ${ }_{\Lambda_{c}}^{209} \mathrm{Bi}$ charmed hypernuclei, in Fig. 1. Charmed nuclei between ${ }_{\Lambda_{c}}^{17} \mathrm{~F}$ and ${ }_{\Lambda_{c}}^{57} \mathrm{Cu}$ are found more bound than their core nuclei, for DFNSC97a-C, in contrast with the two other interactions. This is due to the more attractive nature of DF-NSC97a$\mathrm{C}$ (as the interactions depend on the densities, differences are $3 \mathrm{MeV}$ for $1 \mathrm{~s}$ channel of ${ }_{\Lambda_{c}^{5}}^{5} \mathrm{Li}$ and $5 \mathrm{MeV}$ for $1 \mathrm{~s}$ channel of ${ }_{\Lambda_{c}}^{133} \mathrm{Sb}$ ). The binding energy differences for DF-NSC97f$\mathrm{C}$ are of intermediate values, between DF-NSC97a-C and DF-NSC89-C. It also implies a slightly stronger bound of ${ }_{\Lambda_{c}}^{17} \mathrm{~F}$ with respect to ${ }^{16} \mathrm{O}$, in the case of DF-NSC97f-C.

In the case of $\Lambda_{c}$ separation energies, shown in Fig. 1(b), the general behavior as a function of $\mathrm{A}$ is similar for all force sets, with a maximum difference of $6 \mathrm{MeV}$ between DF-NSC89-C and DF-NSC97a-C. The maximum $\Lambda_{c}$ separation energies are predicted to occur between ${ }_{\Lambda_{c}}^{17} \mathrm{~F}$ and ${ }_{\Lambda_{c}}^{41} \mathrm{Sc}$. Despite the decreasing trend for the $\mathrm{A} \geq 41$ region, $\Lambda_{c}$ separation energies indicate possible bound $\Lambda_{c}$ hypernuclei up to ${ }_{\Lambda_{c}}^{209} \mathrm{Bi}$. This is in contrast with Ref. [27], where its $\mathrm{N}-\Lambda_{c}$ interaction (calculated from HAL QCD approach) predicts the existence of $\Lambda_{c}$ hypernuclei only up to $A=58$. The results of Ref. [25] are also shown in Fig. 1(b) for comparison, where minimum and maximum limits are obtained from QMF-NK3C' and QMF-NK3C, respectively. The main difference between QMF-NK3C' and QMF-NK3C is that the coupling constants of $\mathrm{QMF}-\mathrm{NK} 3 \mathrm{C}^{\prime}$ are obtained from the HAL QCD collaboration, while the coupling constants of QMF-NK3C are obtained from the naive quark counting rule, see Ref. [25]. Fig. 1(b) shows that our results are compatible with these predictions.

The $\Lambda_{c}$, neutron and proton Fermi levels are displayed in Fig. 2. As expected, small (order of keV) differences are observed on the proton and neutron Fermi levels for all force sets. However, there is an average $5 \mathrm{MeV}$ difference on $\Lambda_{c}$ Fermi energies between DF-NSC89-C and DFNSC97a-C, and $2 \mathrm{MeV}$ difference between DF-NSC89-C and DF-NSC97f-C. Due to the Coulomb repulsion, the most bound charmed hypernucleus is ${ }_{\Lambda_{c}}^{17} \mathrm{~F}$, where the energy of the 1s state is $-7 \mathrm{MeV}$ for DF-NSC89-C, -12 $\mathrm{MeV}$ for DF-NSC97a-C and $-8 \mathrm{MeV}$ for DF-NSC97f-C. Since ${ }_{\Lambda_{c}}^{17} \mathrm{~F}$ is predicted with the lowest value for the binding energy, an unambiguous signature of the existence of charmed hypernucleus may be found by producing this system. For charmed nuclei heavier than ${ }_{\Lambda_{c}}^{17} \mathrm{~F}$, the binding energy decreases, resulting in less bound $\Lambda_{c}$ states, due to the Coulomb repulsion.

In order to investigate in more details this behavior, the contributions of the kinetic energy, $\mathrm{N}-\Lambda_{c}$ interaction and the Coulomb potential, to the $\Lambda_{c}$ separation energy are shown in Fig. 3. For all charmed nuclei, DF-NSC97a$\mathrm{C}$ predicts a more bound system, compared to other interactions $\left(\Lambda_{c}\right.$ Total on Fig. 3). The Coulomb repulsion has a increasing behavior proportional to the mass number, while the $\mathrm{N}-\Lambda_{c}$ interaction saturates around ${ }_{\Lambda_{c}}^{57} \mathrm{Cu}$ for all force sets. These effects explain the smaller separation energies in heavier charmed nuclei than ${ }_{\Lambda_{c}}^{41} \mathrm{Sc}$. The saturation property of $\mathrm{N}-\Lambda_{c}$ interactions is compatible with
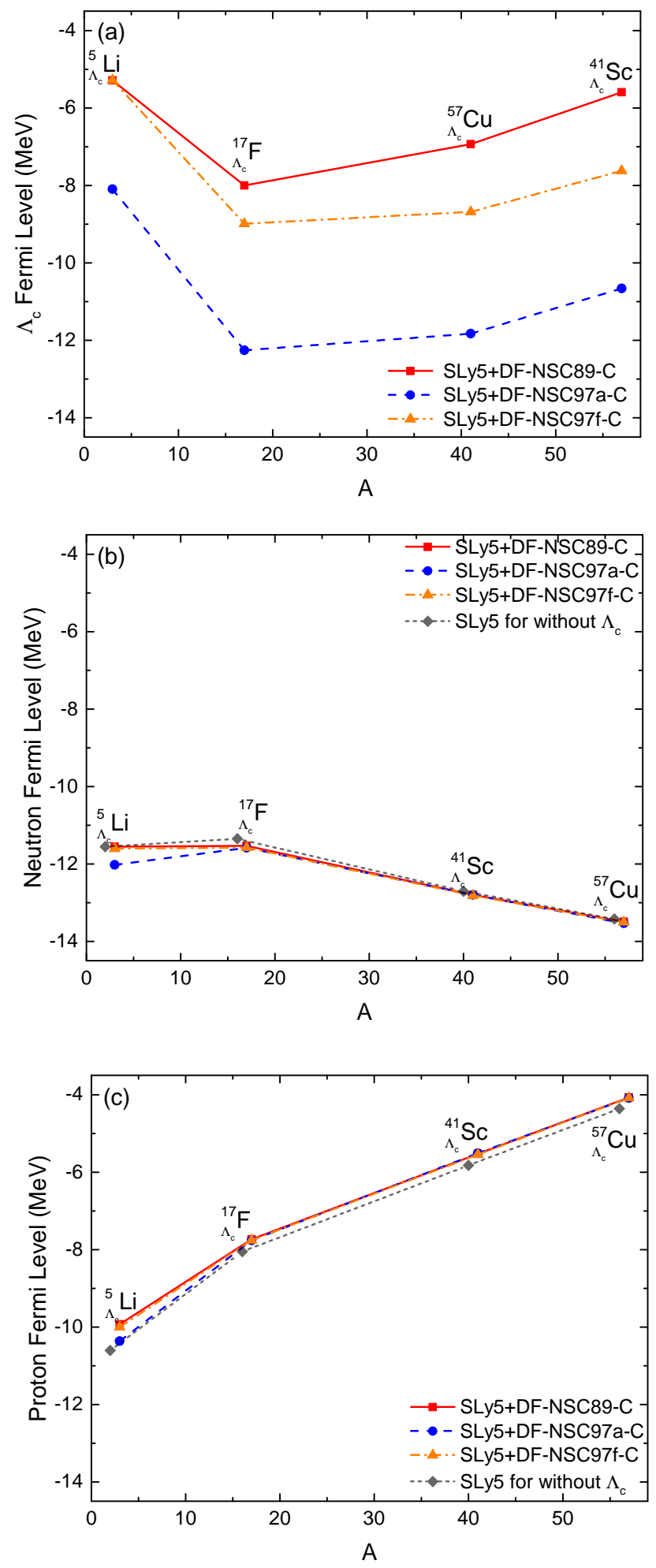

FIG. 2: The Fermi energies of (a) $\Lambda_{c}$, (b) neutrons and (c) protons for DF-NSC89-C, DF-NSC97a-C and DF-NSC97f-C force sets. 

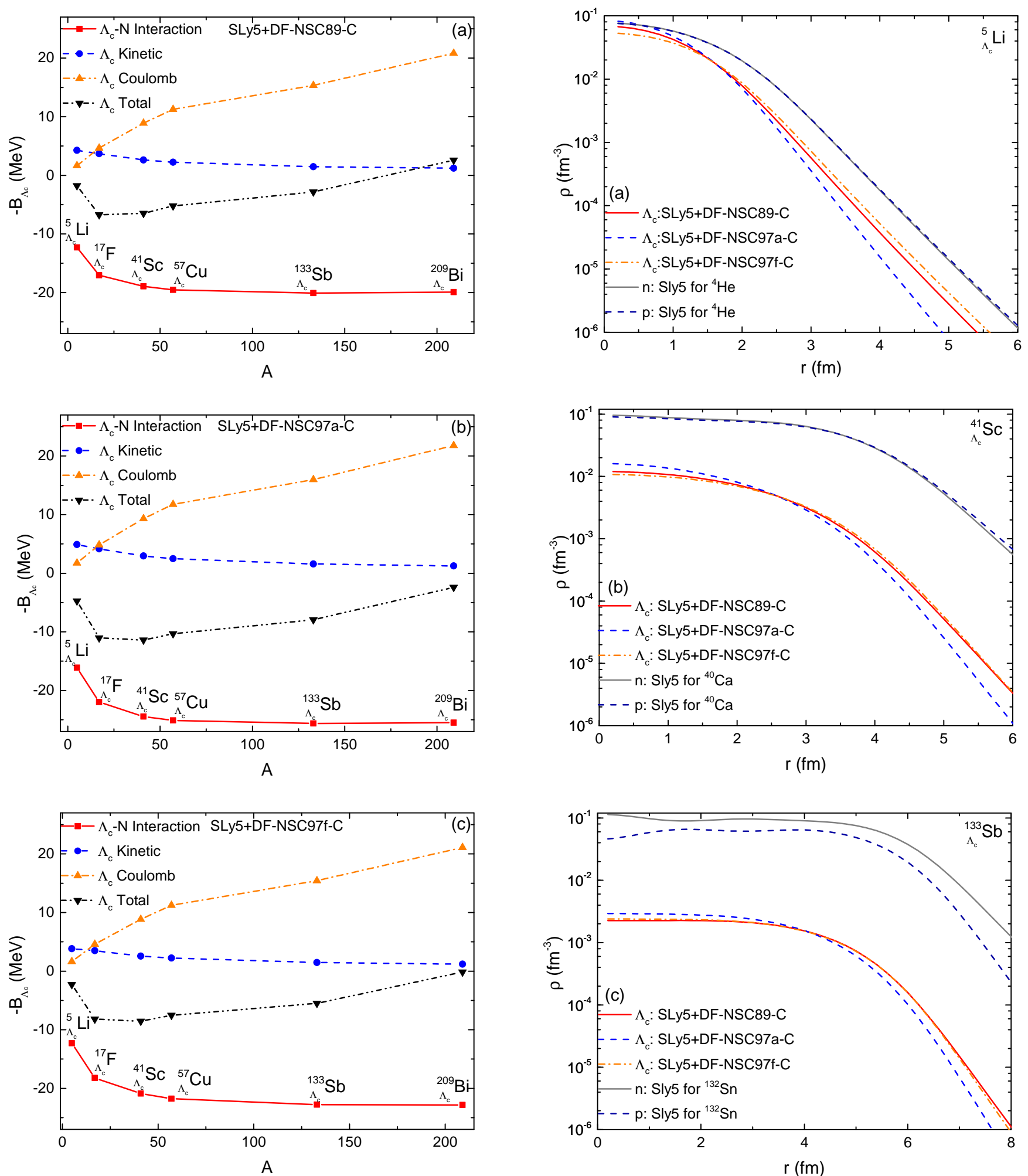

FIG. 3: Contributions of the kinetic energy, N- $\Lambda_{c}$ interaction and the Coulomb potential on the $\Lambda_{c}$ separation energy for (a) DF-NSC89-C, (b)

DF-NSC97a-C and (c) DF-NSC97f-C force sets.

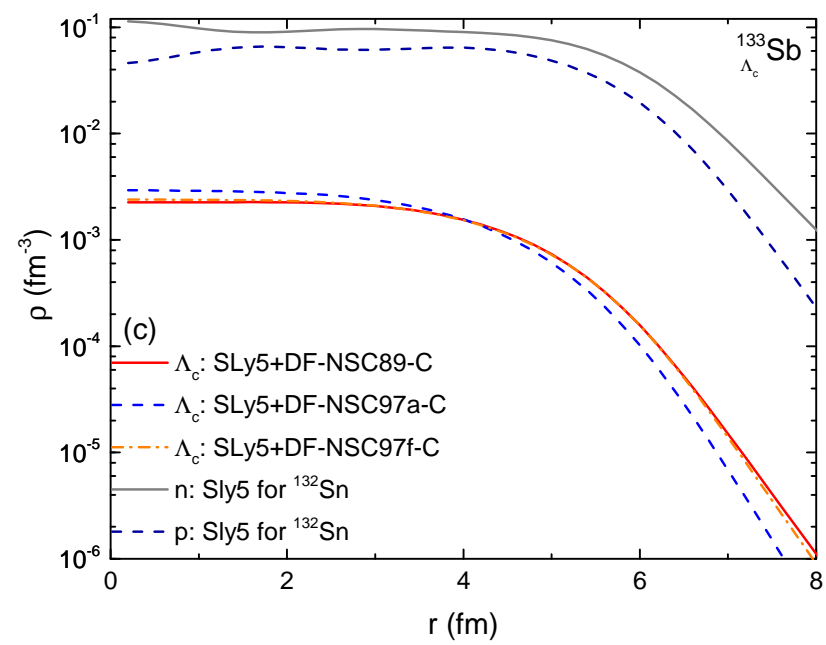

FIG. 4: The $\Lambda_{c}$ density distribution of (a) ${ }_{\Lambda_{c}^{5} \mathrm{Li}}$, (b) ${ }_{\Lambda_{c}}^{41} \mathrm{Sc}$ and (c) ${ }_{\Lambda_{c}}^{133} \mathrm{Sb}$ for DF-NSC89-C, DF-NSC97a-C and DF-NSC97f-C force sets. 
the results of HAL QCD for the $\mathrm{A}>50$ region [27]. However, the present $\mathrm{N}-\Lambda_{c}$ interactions are attractive enough to create bound $\Lambda_{c}$ hypernuclei in the heavy mass region. Due to the saturation behavior of N- $\Lambda_{c}$, Ref. [27] suggests that only light or medium-mass charmed hypernuclei could really exist. This is also in contrast with the work of Vidaña et al. and Haidenbauer et al. (see Refs. $[26,29]$ for details) where stronger $\mathrm{N}-\Lambda_{c}$ attraction were found, as in our case.

In order to investigate the spatial properties of charmed hypernuclei, $\Lambda_{c}$ density distributions are displayed in Fig. 4, together with the proton and neutron distributions of the core nuclei. For all charmed hypernuclei, DF-NSC97a-C generates more packed distributions in the centre of the nucleus, especially for the lightest case $\left(\Lambda_{c}^{5} \mathrm{Li}\right)$. Due to the Coulomb repulsion, the $\Lambda_{c}$ distribution extends to larger radii, in heavier hypernuclei. This dilution of the charmed baryon, in the nucleus, impacts its density at the centre, which can decrease by a couple of orders of magnitude, from the lightest to the heaviest charmed hypernuclei.

\section{B. Comparison of $\Lambda_{c}$ vs. $\Lambda$ in Hypernuclei}

It could be relevant to compare the properties of hypernuclei and charmed ones, to understand for instance the respective role of the Coulomb interaction as well as of the scaling $N \Lambda_{c}$ interaction factor $K$. On this purpose, $\Lambda$ hypernuclei are calculated with the same HF scheme, using DF-NSC89, DF-NS97a and DF-NSC97f force sets, as introduced in Table I. Starting from ${ }_{\Lambda}^{5} \mathrm{He}$, we compare ${ }_{\Lambda}^{17} \mathrm{O},{ }_{\Lambda}^{41} \mathrm{Ca},{ }_{\Lambda}^{57} \mathrm{Ni},{ }_{\Lambda}^{133} \mathrm{Sn}$ and ${ }_{\Lambda}^{209} \mathrm{~Pb}$ hypernuclei with their charmed counterparts.

We first represent Fermi energies for $\Lambda_{c}$ and $\Lambda$ in Fig. 5 . In the case of the lightest ${ }_{\Lambda_{c}^{5}}^{5} \mathrm{Li}-{ }_{\Lambda}^{5} \mathrm{He}$ pair, Fermi energies are nearly identical, between the charmed and normal NSC force sets. However, both $\Lambda$ and $\Lambda_{c}$ Fermi levels increases (in absolute value), up to the ${ }_{\Lambda}^{17} \mathrm{O}-{ }_{\Lambda_{c}}^{17} \mathrm{Li}$ pair, where the Fermi energy differences between $\Lambda$ and $\Lambda_{c}$ are 5.02 MeV for NSC89, $5.92 \mathrm{MeV}$ for NSC97a and 5.81 $\mathrm{MeV}$ for NSC97f. But, as the mass number increases, larger energy differences occur, due to the Coulomb repulsion, especially after the $\mathrm{A}=17$ region. This leads to unbound state for ${ }_{\Lambda_{c}}^{209} \mathrm{Bi}$ charmed hypernucleus for DFNSC89-C and DF-NSC97f-C. The increasing effect of the Coulomb repulsion is also the main reason why ${ }_{\Lambda_{c}}^{17} \mathrm{~F}$ is an excellent test nucleus, to probe charmed hypernuclei: the effect of the $\mathrm{N}-\Lambda_{c}$ interaction is expected to be noticeable and therefore could be probed in order to constrain it. It should be noted that DF-NSC97a-C has a large enough attraction, to counter balance the Coulomb repulsion, leading to bound ${ }_{\Lambda_{c}}^{209} \mathrm{Bi}$ charmed hypernucleus. Also, the maximum Fermi energy differences are spotted at ${ }_{C}^{209} \mathrm{~Pb}$ ${ }_{\Lambda_{c}}^{209} \mathrm{Bi}$ pair: $26 \mathrm{MeV}$ for NSC89 (and also for NSC97a), and $32 \mathrm{MeV}$ for NSC97f.

Finally, the $\Lambda_{c}(\Lambda)$ single particle energies are displayed in Fig. 6: for a light charmed hypernucleus ${ }_{\Lambda_{c}^{5}}^{5} \mathrm{Li}\left({ }_{\Lambda}^{5} \mathrm{He}\right)$,

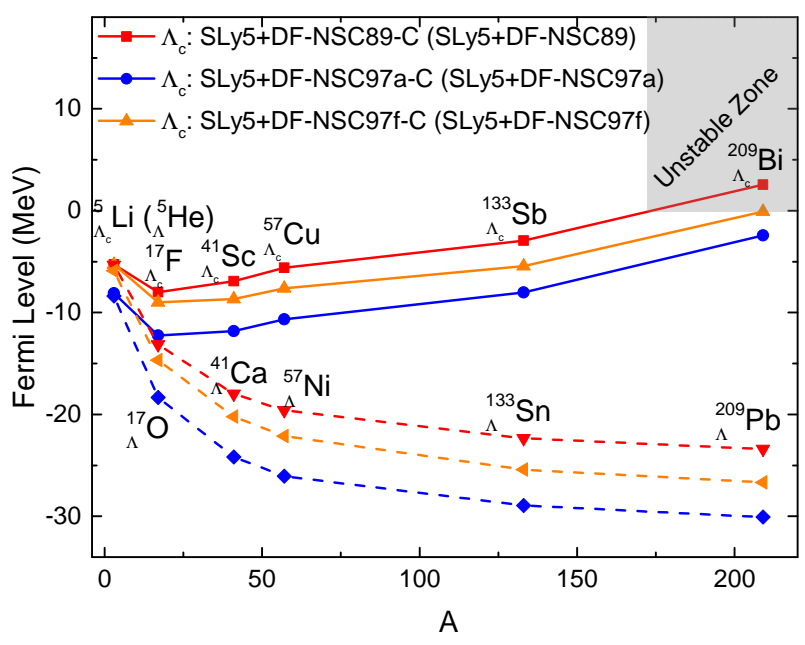

FIG. 5: The Fermi energy of the $\Lambda_{c}(\Lambda)$ for the DF-NSC89-C (DF-NSC89), DF-NSC97a-C (DF-NSC97a) and DF-NSC97f-C (DF-NSC97f) force sets, in solid lines (dashed lines). Unbound hypernuclei are displayed in the gray area (Unstable Zone).

for a medium-mass charmed hypernucleus ${ }_{\Lambda}^{41} \mathrm{Sc}\left({ }_{\Lambda}^{41} \mathrm{Ca}\right)$ and for heavy charmed hypernucleus ${ }_{1}^{133} \mathrm{Sb}\left({ }_{\Lambda}^{133} \mathrm{Sn}\right)$. In the ${ }_{\Lambda_{c}^{5}}^{5} \mathrm{Li}$ case, DF-NSC89-C and DF-NSC97f-C predict similar values for the 1s state. However, DF-NSC97a-C differs by $2.72 \mathrm{MeV}$ from DF-NSC89-C and DF-NSC97fC. Since the Coulomb repulsion is rather weak for the case of ${ }_{\Lambda_{c}^{5}}^{5} \mathrm{Li}$, similar results are obtained in the case of hypernuclei: in ${ }_{\Lambda}^{5} \mathrm{He}$, there is $0.59 \mathrm{MeV}$ difference between DF-NSC89 and DF-NSC97f, but $3.10 \mathrm{MeV}$ between DFNSC89 and DF-NSC97a. In addition, DF-NSC89-C and DF-NSC97f-C do not allow for any excited state in the $\Lambda_{c}^{5} \mathrm{Li}$ case, whereas DF-NSC89a-C allows for such a state.

The situation starts to change for the ${ }_{\Lambda}^{41} \mathrm{Sc}$ case, where DF-NSC89-C and DF-NSC97f-C differ from each other, by $1.76 \mathrm{MeV}$ for the $1 \mathrm{~s}$ state. The gap, between $1 \mathrm{~s}$ and $1 \mathrm{p}$ states, is $3.93 \mathrm{MeV}$ for DF-NSC89-C, $4.43 \mathrm{MeV}$ for DF-NSC97f-C and $4.68 \mathrm{MeV}$ for DF-NSC97a-C. DFNSC89-C and DF-NSC97f-C allow only 1p state while DF-NSC97a-C also allows for a 1d state in addition. Therefore, a possible spectroscopy of charmed hypernuclei, could allow to disentangle between the various interations. In the case of ${ }_{\Lambda}^{41} \mathrm{Ca}$, 1s state energy levels more bound because of the Coulomb repulsion: such energy differences on the 1s state are $11.04 \mathrm{MeV}$ for NSC89, 17.02 MeV for NSC-97a and 11.54 MeV for NSC-97f. In the ${ }_{\Lambda}^{133} \mathrm{Sb}$ case, the predicted levels are different for each force set: charmed nuclei are becoming less bound due to the Coulomb repulsion. An average of $20 \mathrm{MeV}$ difference is predicted for the $1 \mathrm{~s}$ state between ${ }^{133} \mathrm{Sn}$ and ${ }_{\Lambda}^{133} \mathrm{Sb}$. These results are in agreement with those previously obtained by RMF and QMC models [20, 25]. More precisely, the present $\Lambda_{c}$ 1s state predictions are located between those of QMF-NK3C and QMF-NK3C' [25], for 

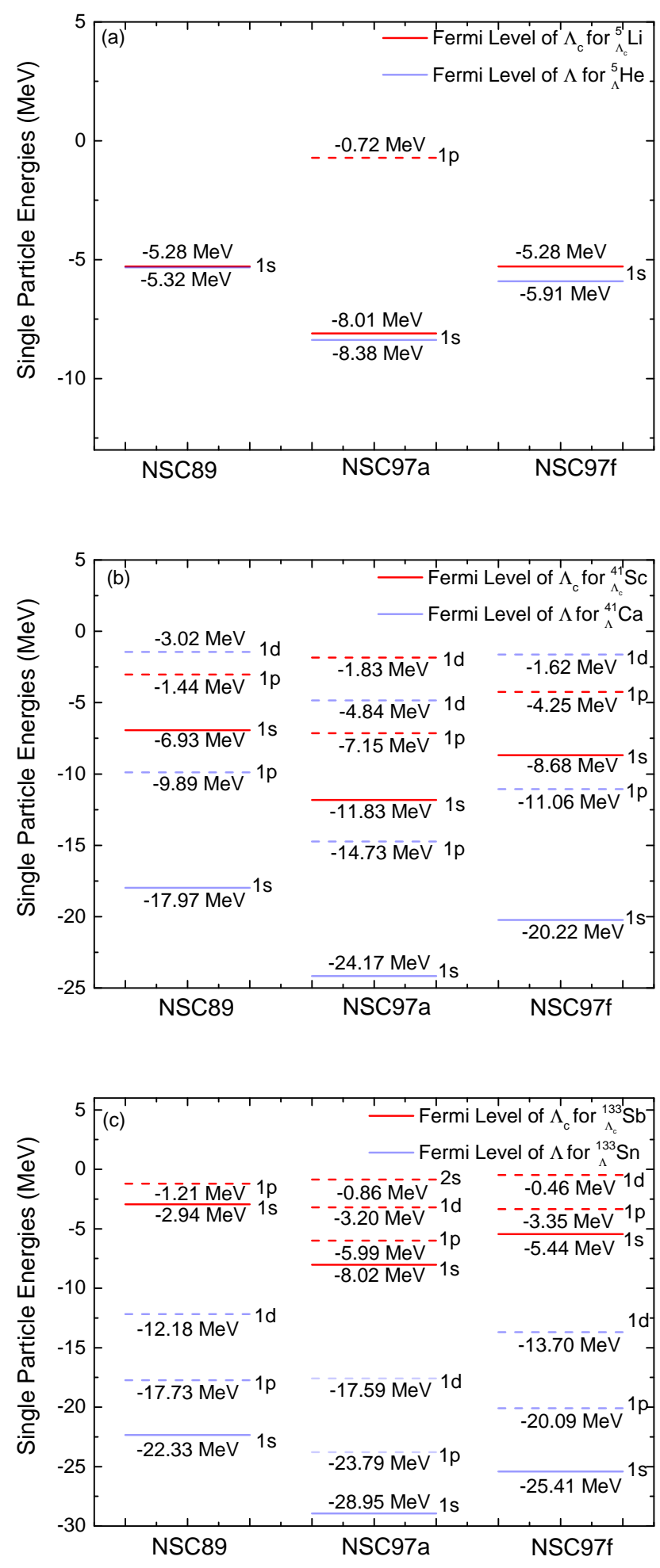

FIG. 6: The $\Lambda_{c}(\Lambda)$ single particle spectrum of ${ }_{\Lambda_{c}^{5}}^{5} \mathrm{Li}$ $\left({ }_{\Lambda}^{5} \mathrm{He}\right)(\mathrm{a}),{ }_{\Lambda}^{41} \mathrm{Sc}\left({ }_{\Lambda}^{41} \mathrm{Ca}\right)(\mathrm{b})$ and ${ }_{\Lambda}^{133} \mathrm{Sb}\left({ }_{\Lambda}^{133} \mathrm{Sn}\right)(\mathrm{c})$ for NSC89, NSC97a and NSC97f force sets.

all charmed hypernuclei.

\section{CONCLUSIONS}

In this work we have investigated the ground state properties of charmed hypernuclei, close to doubly magic closed-shell nuclei. We use SLy5 Skyrme force for the NN interaction and we generate $\mathrm{N} \Lambda_{c}$ interactions from the $\mathrm{N} \Lambda$ interactions obtained from microscopic BruecknerHartree-Fock calculations.

Since $\Lambda_{c}$ is positively charged, the Coulomb interaction plays an important role in the ground state properties of charmed hypernuclei. Additionally, it is shown that $\mathrm{N} \Lambda_{c}$ interactions have a saturation property around ${ }_{\Lambda_{c}}^{57} \mathrm{Cu}$. Due to these reasons, $\Lambda_{c}$ is maximally bound for mass numbers between ${ }_{\Lambda_{c}}^{17} \mathrm{~F}$ and ${ }_{\Lambda_{c}}^{41} \mathrm{Sc} .{ }_{\Lambda_{c}}^{17} \mathrm{~F}$ is an excellent candidate for the experimental measurements of charmed hypernuclei, since the predictions from the different functionals considered in this study are the largest one and the most different for this system. For charmed hypernuclei heavier than ${ }_{\Lambda_{c}}^{41} \mathrm{Sc}$, Coulomb repulsion becomes comparable in absolute value with the attractive $\mathrm{N} \Lambda_{c}$ interactions, and charmed hypernuclei become less and less bound as $A$ increases, leading to unbound ${ }_{\Lambda_{c}}^{209} \mathrm{Bi}$ (for DFNSC89-C and DF-NSC97f-C interactions). Our results confirm previous ones obtained by the HAL QCD collaboration: ${ }_{\Lambda_{c}}^{209} \mathrm{Bi}$ is unbound and the $\mathrm{N} \Lambda_{c}$ interaction saturates in the mid- $A$ region. However, DF-NSC97a-C has a strong enough attraction to counter balance the Coulomb repulsion, leading to weakly-bound ${ }_{\Lambda_{c}}^{209} \mathrm{Bi}$ charmed hypernucleus.

In addition, the gap between $1 \mathrm{~s}$ and $1 \mathrm{~d}$ states is found to be about $4 \mathrm{MeV}$ for the majority of charmed hypernuclei. As a final conclusion, the reason of the uncertainties in the $\mathrm{N} \Lambda_{c}$ interaction is mostly due to the lack of experimental measurements. Our results, confronted to others, predict different behavior for charmed hypernuclei as function of the mass number $A$, for which the future experimental facilities will help in selecting among the best density functional approaches.

\section{ACKNOWLEDGMENT}

This work is supported by the Scientific and Technological Research Council of Turkey (TÜBİTAK) under project number MFAG-118F098 and the Yildiz Technical University under project number FBI-2018-3325 and FBA-2021-4229. E.K. and J.M. are both supported by the CNRS/IN2P3 NewMAC project, and are also grateful to PHAROS COST Action MP16214. J.M. is grateful to the LABEX Lyon Institute of Origins (ANR-10LABX-0066) of the Université de Lyon for its financial support within the program Investissements d'Avenir (ANR-11-IDEX-0007) of the French government operated by the National Research Agency (ANR). 
[1] C. L. Aubert et al., Phys. Rev. Lett. 33, 1404 (1974).

[2] J. Augustin et al., Phys. Rev. Lett. 33, 1406 (1974).

[3] E. G. Cazzoli et al., Phys. Rev. Lett. 34, 1125 (1975).

[4] G. Goldhaber et al., Phys. Rev. Lett. 37, 255 (1976).

[5] I. Peruzzi et al., Phys. Rev. Lett. 37, 569 (1976).

[6] B. Knapp et al., Phys. Rev. Lett. 37, 882 (1976).

[7] M. Danysz, and J. Pniewski, Philos. Mag. Ser. 5 44, 348 (1953).

[8] C. B. Dover and S. H. Kahana, Phys. Rev. Lett. 39, 1506 (1977).

[9] Y. A. Batusov et al., JINR Preprint E1-10069 (JINR, Dubna 1976).

[10] Y. A. Batusov et al., JETP Lett. 33, 56 (1981).

[11] V. V. Lyukov, Nuovo Cimento A 102, 583 (1989).

[12] J. Riedl, A. Schafer, and M. Stratmann, Eur. Phys. J. C 52, 987 (2007).

[13] R. Shyam and K. Tsushima, Phys. Lett. B 770, 236 (2017).

[14] G. Krein, A. W. Thomas, and K. Tsushima, Prog. Part. Nucl. Phys. 100, 161 (2018).

[15] G. Bhamathi, Phys. Rev. C 24, 1816 (1981).

[16] H. Bando and M. Bando, Phys. Lett. B 109, 164 (1982).

[17] H. Bando and S. Nagata, Prog. Theor. Phys. 69, 557 (1983).

[18] B. F. Gibson, C. B. Dover, G. Bhamathi, and D. R. Lehman, Phys. Rev. C 27, 2085 (1983).

[19] H. Bando, Prog. Theor. Phys. 81, 197 (1985).

[20] Y. H. Tan and P. Z. Ning, Europhys. Lett. 67, 355 (2004).

[21] Y. H. Tan, X. H. Zhong, C. H. Cai, and P. Z. Ning, Phys. Rev. C 70, 054306 (2004).

[22] K. Tsushima and F. C. Khanna, Prog. Theor. Phys. Suppl. 149, 160 (2003).

[23] K. Tsushima and F. C. Khanna, Phys. Rev. C 67, 015211 (2003).

[24] K. Tsushima and F. C. Khanna, J. Phys. G 30, 1765 (2004).
[25] L. Wu, J. Hu, and H. Shen, Phys. Rev. C 101, 024303 (2020).

[26] I. Vidaña, A. Ramos, and C. E. Jiménez-Tejero, Phys. Rev. C 99, 045208 (2019).

[27] T. Miyamoto et al., Nucl. Phys. A 971, 113 (2018).

[28] J. Haidenbauer and G. Krein, Eur. Phys. J. A 54, 199 (2018).

[29] J. Haidenbauer, A. Nogga and I. Vidaña, Eur. Phys. J. A 56, 195 (2020).

[30] H.-J. Schulze and T. Rijken, Phys. Rev. C 88, 024322, (2013).

[31] H. Güven, K. Bozkurt, E. Khan, and J. Margueron, Phys. Rev. C 98, 014318 (2018).

[32] I. Vidaña, A. Polls, A. Ramos and H.-J. Schulze, Phys. Rev. C 64, 044301 (2001)

[33] N. I. Starkov and V. A. Tsarev, Nuclear Physics A 450, 507c (1986).

[34] S. Perez-Martin and L. M. Robledo, Phys. Rev. C 78, 014304 (2008).

[35] J. Cugnon, A. Lejeune and H.-J.Schulze, Phys. Rev. C 62, 064308 (2000).

[36] M. Bender, P.H. Heenen, P.-G. Reinhard, Rev. Mod. Phys. 75, 121 (2003).

[37] O. Hashimoto and H. Tamura, Progress in Particle and Nuclear Physics 57, 564-653 (2006).

[38] T. Motoba, D. E. Lanskoyb, D. J. Millener, and Y. Yamamoto, Nucl. Phys. A804, 99-115 (2008).

[39] E. Khan, J. Margueron, F. Gulminelli, and Ad. R. Raduta, Phys. Rev. C 92, 044313 (2015).

[40] G. Chanfray and J. Margueron, Phys. Rev. C 102, 024331 (2020).

[41] M. Tanabashi et al. (Particle Data Group), Phys. Rev. D 98, 030001 (2019).

[42] J. Margueron, E. Khan and F. Gulminelli, Phys. Rev. C 96, 054317 (2017).

[43] J. Bartel, K. Bencheikh, and J. Meyer, Phys. Rev. C 77, 024311 (2008).

[44] P. Ring and P. Schuck, The Nuclear Many-Body Problem (Springer-Verlag, Berlin, 1980). 